May 2021

\title{
Divining Structural Factors Related to Intervention Success or Failure: Cultural Sexism versus Other Macro-Level Factors
}

Blair T. Johnson

University of Connecticut, blair.t.johnson@uconn.edu

Christine M. Curley

christine.curley@uconn.edu

Follow this and additional works at: https://opencommons.uconn.edu/chip_docs

Part of the Child Psychology Commons, Clinical Psychology Commons, Counseling Psychology Commons, Developmental Psychology Commons, Development Studies Commons, Educational Psychology Commons, Educational Sociology Commons, Education Policy Commons, Family, Life Course, and Society Commons, Gender and Sexuality Commons, Health and Physical Education Commons, Health Psychology Commons, Human Ecology Commons, Inequality and Stratification Commons, Place and Environment Commons, School Psychology Commons, Social Policy Commons, Social Welfare Commons, and the Sociology of Culture Commons

\section{Recommended Citation}

Johnson, Blair T. and Curley, Christine M., "Divining Structural Factors Related to Intervention Success or Failure: Cultural Sexism versus Other Macro-Level Factors" (2021). CHIP Documents. 38.

https://opencommons.uconn.edu/chip_docs/38 


\section{Technical Summary}

\section{Divining Structural Factors Related to Intervention Success or Failure: Cultural Sexism versus Other Macro-Level Factors}

by

Blair T. Johnson and Christine M. Curley

University of Connecticut

Table of Contents

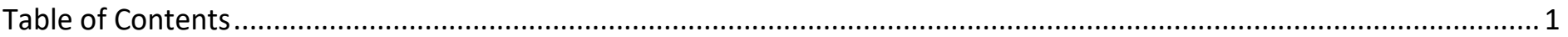

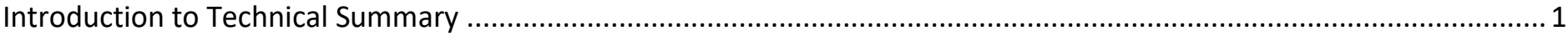

Cultural Sexism, Cultural Tightness, and History of Slave-Holding ........................................................................ 1

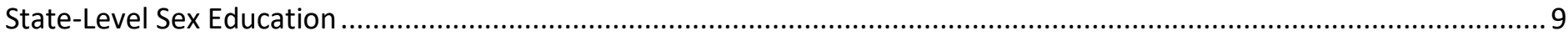

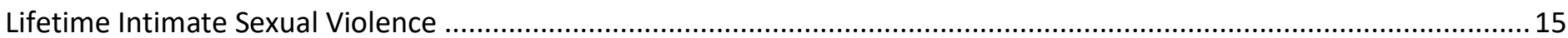

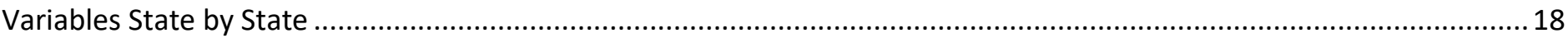

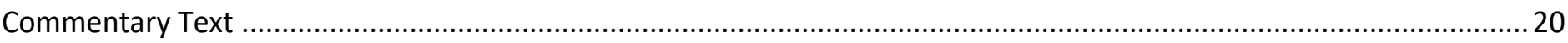

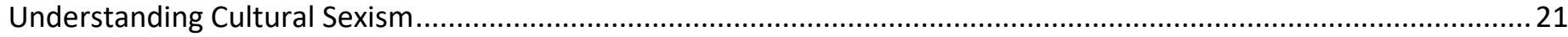

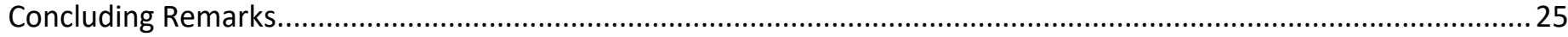

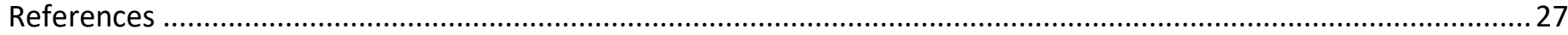

\section{Introduction to Technical Summary}

We wrote this technical summary is to accompany a commentary that the editors of Clinical Psychology: Science and Practice (CP:SP) invited regarding a spatial meta-analysis authored by Price and colleagues (in press). For this summary, the District of Columbia (DC) was assumed to be a state. Analyses were undertaken in Stata 16.1. The Stata database is also available in this archive, named cultural_sexism_29apr2021.dta. The final section gives the text of the commentary, but see the published article for the final version.

\section{Cultural Sexism, Cultural Tightness, and History of Slave-Holding}




\section{Technical Summary}

Descriptive summary of variables:

. sum csexism hasint loosenessstiffness pop2010 gini pctslaves1860

\begin{tabular}{|c|c|c|c|c|c|c|c|}
\hline Variable & \multicolumn{2}{|c|}{ Obs } & Mean & \multicolumn{2}{|c|}{ Std. Dev. } & Min & Max \\
\hline csexism & 51 & \multicolumn{2}{|c|}{.0003922} & \multicolumn{2}{|c|}{.992935} & -2.61 & 1.9 \\
\hline hasint & 51 & \multicolumn{2}{|c|}{.627451} & \multicolumn{2}{|c|}{.4882944} & 0 & 1 \\
\hline loosenesss s & 50 & \multicolumn{2}{|c|}{50.1388} & \multicolumn{2}{|c|}{12.59583} & 27.37 & 78.86 \\
\hline pop2010 & 51 & \multicolumn{2}{|c|}{6053834} & \multicolumn{2}{|c|}{6823984} & 563626 & $3.73 e+07$ \\
\hline gini & 51 & \multicolumn{2}{|c|}{.4661647} & \multicolumn{2}{|c|}{.0234549} & .4063 & .542 \\
\hline pctslav 1860 & 51 & \multicolumn{2}{|c|}{9.502745} & \multicolumn{2}{|c|}{17.13809} & 0 & 57.2 \\
\hline Variable & Obs & Mean & Std. & Dev. & Min & $\operatorname{Max}$ & \\
\hline csexism & 51 & .0003922 & .992 & 935 & -2.61 & 1.9 & \\
\hline hasint & 51 & .627451 & .4882 & 944 & 0 & 1 & \\
\hline loosenesss s & 50 & 50.1388 & $12.5 \mathrm{~s}$ & 583 & 27.37 & 78.86 & \\
\hline pop2010 & 51 & 6053834 & 6823 & 984 & 563626 & $3.73 e+07$ & \\
\hline gini & 51 & .4661647 & .0234 & 549 & .4063 & .542 & \\
\hline
\end{tabular}

Note. DC is missing a value for Looseness-Tightness. csexism=cultural sexism. has int=has intervention (1 vs. not, 0$)$. loosenesstiffness=Looseness-Tightness. pop2010=population in 2010 census. gini=income inequality in 2010 census. pctslaves1860=percentage of slaves of total population in state or territory in 1860 census (missing values converted to zeroes).

Correlation matrix:

- pwcorr csexism hasint loosenessstiffness pop2010 gini pctslaves1860, star(.05)

\begin{tabular}{r|cccccc} 
& csexism & hasint loosen s & pop2010 & gini pct 1860 \\
\hline csexism & 1.0000 & & & & & \\
hasint & -0.1948 & 1.0000 & & & & \\
loosenesss s & $0.7608^{*}$ & -0.1983 & 1.0000 & & & \\
pop2010 & -0.0190 & $0.4360^{*}$ & -0.0706 & 1.0000 & & \\
gini & -0.1094 & 0.0219 & 0.2249 & $0.3936 *$ & 1.0000 & \\
pctslav 1860 & $0.4765^{*}$ & 0.0044 & $0.6569 *$ & 0.1623 & $0.3487^{*}$ & 1.0000
\end{tabular}

Note. P-values do not weight for population size. If you do, then:

. pwcorr csexism hasint loosenessstiffness pop2010 gini pctslaves1860 [aw=pop2010], star(.05)

\begin{tabular}{r|cccccc} 
& csexism & hasint loosen s & pop2010 & gini pct 1860 \\
\hline csexism & 1.0000 & & & & & \\
hasint & -0.2285 & 1.0000 & & & & \\
loosenesss s & $0.7864^{*}$ & -0.2431 & 1.0000 & & & \\
pop2010 & -0.2478 & $0.3801^{*}$ & $-0.4083^{*}$ & 1.0000 & & \\
gini & -0.1641 & 0.1588 & -0.1466 & $0.5184^{*}$ & 1.0000 & \\
pctslav 1860 & $0.6203^{*}$ & -0.0718 & $0.6420^{*}$ & -0.0488 & 0.1506 & 1.0000
\end{tabular}

Note. Weight is 2010 population, pop2010. Some values for conventional statistical significance change. 


\section{Technical Summary}

One significant correlation was hasint•pop2010, such that states with interventions are far larger in population:

\begin{tabular}{r|cccccc}
$\begin{array}{l}\text { Number of obs } \\
\text { Root MSE }\end{array}$ & $\begin{array}{r}51 \\
6.2 \mathrm{e}+06\end{array}$ & $\begin{array}{l}\text { R-squared } \\
\text { Adj R-squared }=\end{array}$ & 0.1901 \\
Source & Partial SS & df & MS & F & Prob >F \\
\hline Model & $4.425 \mathrm{e}+14$ & & 1 & $4.425 \mathrm{e}+14$ & 11.50 & 0.0014 \\
hasint & $4.425 \mathrm{e}+14$ & & 1 & $4.425 \mathrm{e}+14$ & 11.50 & 0.0014 \\
Residual & $1.886 \mathrm{e}+15$ & & 49 & $3.849 \mathrm{e}+13$ & & \\
\hline Total & $2.328 \mathrm{e}+15$ & & 50 & $4.657 \mathrm{e}+13$ & &
\end{tabular}

\begin{tabular}{|c|c|c|c|}
\hline \multirow[b]{2}{*}{ hasint } & \multicolumn{3}{|c|}{ Summary of pop 2010} \\
\hline & Mean & Std. Dev. & Freq. \\
\hline$\theta$ & 2230938.5 & 1477381.7 & 19 \\
\hline 1 & 8323678.3 & 7717813.5 & 32 \\
\hline Total & 6053834.1 & 6823984.3 & 51 \\
\hline
\end{tabular}

States with interventions are 3.731021 times larger in population, on average.

One focus of the commentary is confounds of cultural sexism with other structural variables, the correlation matrix shows that the single largest correlation (by far, $r=.76$ ) is the one of sexism with looseness-tightness.

As the following figure shows, states without interventions (open symbols in green) appear not to be outliers around the regression lines, relative to states with interventions (solid symbols in navy blue).

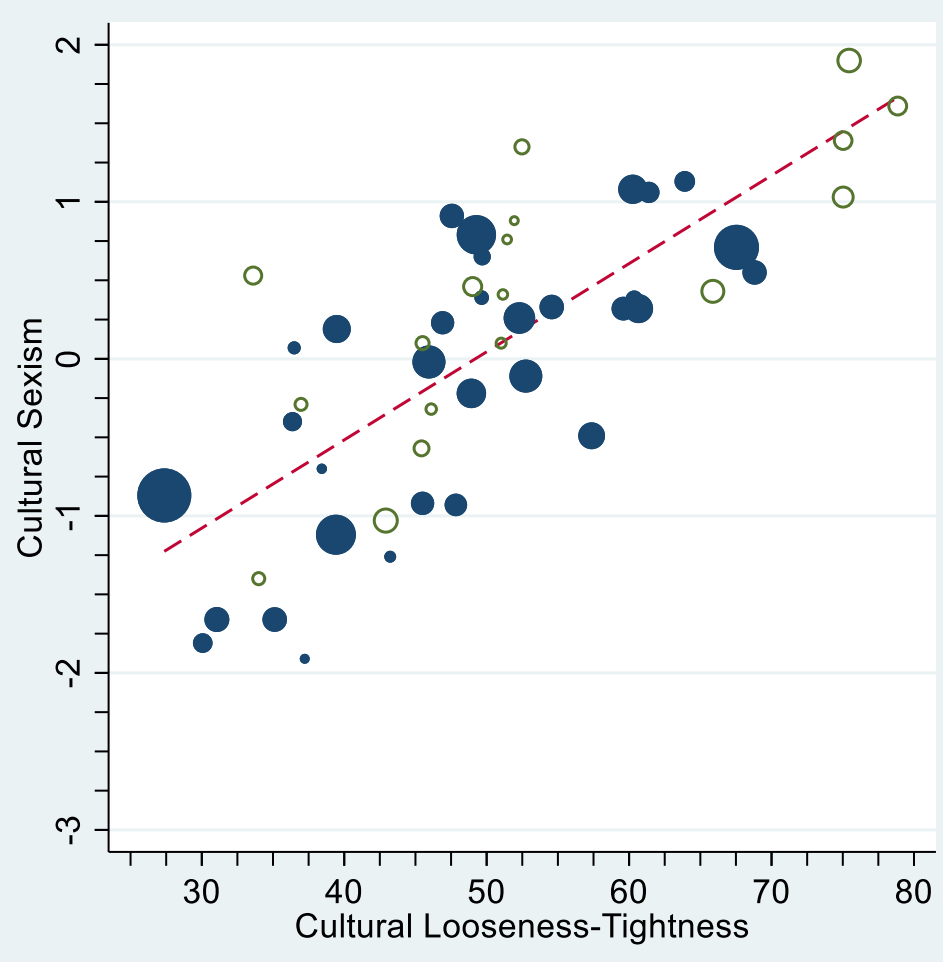

Command: 


\section{Technical Summary}

twoway (Ifit csexism loosenessstiffness, Ipattern(dash) Icolor(cranberry)) (scatter csexism loosenessstiffness [aw=pop2010] if hasint==1, msymbol(O) mcolor(navy) msize(*.33)

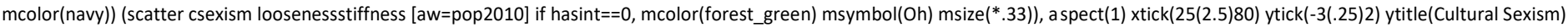
xtitle(Cultural Looseness-Tightness) legend(off)

And a test evaluating the residuals confirms that assumption.

- anova csexlooseres hasint [aw=pop2010]

(sum of wgt is $3.0814 \mathrm{e}+08$ )

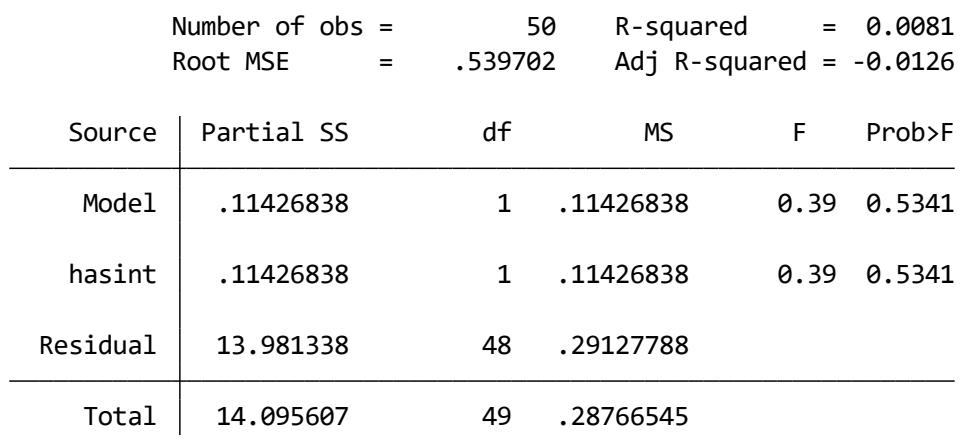

. tab hasint [aw=pop2010], sum(csexlooseres)

\begin{tabular}{r|rrrr}
\multirow{2}{*}{ hasint } & \multicolumn{4}{|c}{ Summary of Residuals } \\
& Mean & Std. Dev. & Freq. & Obs. \\
\hline 0 & .12069651 & .60711917 & 41786108 & 18 \\
1 & -.01893483 & .52683563 & 266357707 & 32 \\
\hline Total & $9.368 \mathrm{e}-10$ & .53634452 & 308143815 & 50
\end{tabular}

Yet, residuals are correlated with the cultural sexism factor (see bottom row):

- pwcorr hasint pop2010 csexism loosenessstiffness csexlooseres [aw=pop2010], star(.05)

\begin{tabular}{r|ccccc} 
& hasint & pop2010 & csexism loosen s csexlo s \\
\hline hasint & 1.0000 & & & & \\
pop2010 & $0.3801^{*}$ & 1.0000 & & & \\
csexism & -0.2285 & -0.2478 & 1.0000 & & \\
loosenesss s & -0.2431 & $-0.4083^{*}$ & $0.7864^{*}$ & 1.0000 & \\
csexlooseres & -0.0900 & 0.1033 & $0.6177^{*}$ & -0.0000 & 1.0000
\end{tabular}

Such that the stricter the culture, the larger the residual. Thus, there is a form of heteroscedasticity in the data for these two variables. 


\section{Technical Summary}

Here are the most extreme residuals, sorted from most negative to most positive (and omitting states in between):

. lis state hasint csexism loosenessstiffness csexlooseres if csexlooseres<-.59| csexlooseres>.62

\begin{tabular}{|c|c|c|c|c|c|}
\hline & state & hasint & csexism & loosen s & csexloo s \\
\hline 1. & VERMONT & 1 & -1.91 & 37.23 & -1.299441 \\
\hline 2. & RHODE ISLAND & 1 & -1.26 & 43.23 & -.9485205 \\
\hline 3. & MASSACHUSETTS & 1 & -1.66 & 35.12 & -.944265 \\
\hline 4. & VIRGINIA & 1 & -.49 & 57.37 & -.8833507 \\
\hline 5. & MINNESOTA & 1 & -.93 & 47.84 & -.8483132 \\
\hline 6. & OREGON & 1 & -1.81 & 30.07 & -.84254 \\
\hline 7. & WASHINGTON & 1 & -1.66 & 31.06 & -.741888 \\
\hline 8. & MARYLAND & 1 & -.92 & 45.5 & -.7216722 \\
\hline 9. & COLORADO & 0 & -1.03 & 42.92 & -.703068 \\
\hline 10. & MAINE & 0 & -1.4 & 34 & -.6284369 \\
\hline 11. & NEW YORK & 1 & -1.12 & 39.42 & -.6186051 \\
\hline 42 . & UTAH & 1 & .65 & 49.69 & .6394708 \\
\hline 43. & NORTH DAKOTA & 0 & .76 & 51.44 & .6622394 \\
\hline 44. & NEW JERSEY & 1 & .19 & 39.48 & .688404 \\
\hline 45 . & HAWAII & 1 & .07 & 36.49 & .7174451 \\
\hline 46. & WYOMING & 0 & .88 & 51.94 & .7573161 \\
\hline 47. & FLORIDA & 1 & .79 & 49.28 & .7999079 \\
\hline 48. & ARIZONA & 1 & .91 & 47.56 & 1.005644 \\
\hline 49 . & WEST VIRGINIA & 0 & 1.35 & 52.48 & 1.200399 \\
\hline 50 . & NEVADA & 0 & .53 & 33.61 & 1.321003 \\
\hline 51. & DISTRICT OF COLUMBIA & 0 & -2.61 & . & . \\
\hline
\end{tabular}

The most negative residuals are in New England (Vermont, Rhode Island, Massachusetts); the most positive are to the south (West Virginia) and west (Arizona, Nevada); two of these states have no interventions.

The following figure shows all observations along with the best-fitting linear line, sizing the markers to be proportional to the population size; solid (navy blue) markers are for states with interventions, those without have hollow (green) markers. 


\section{Technical Summary}

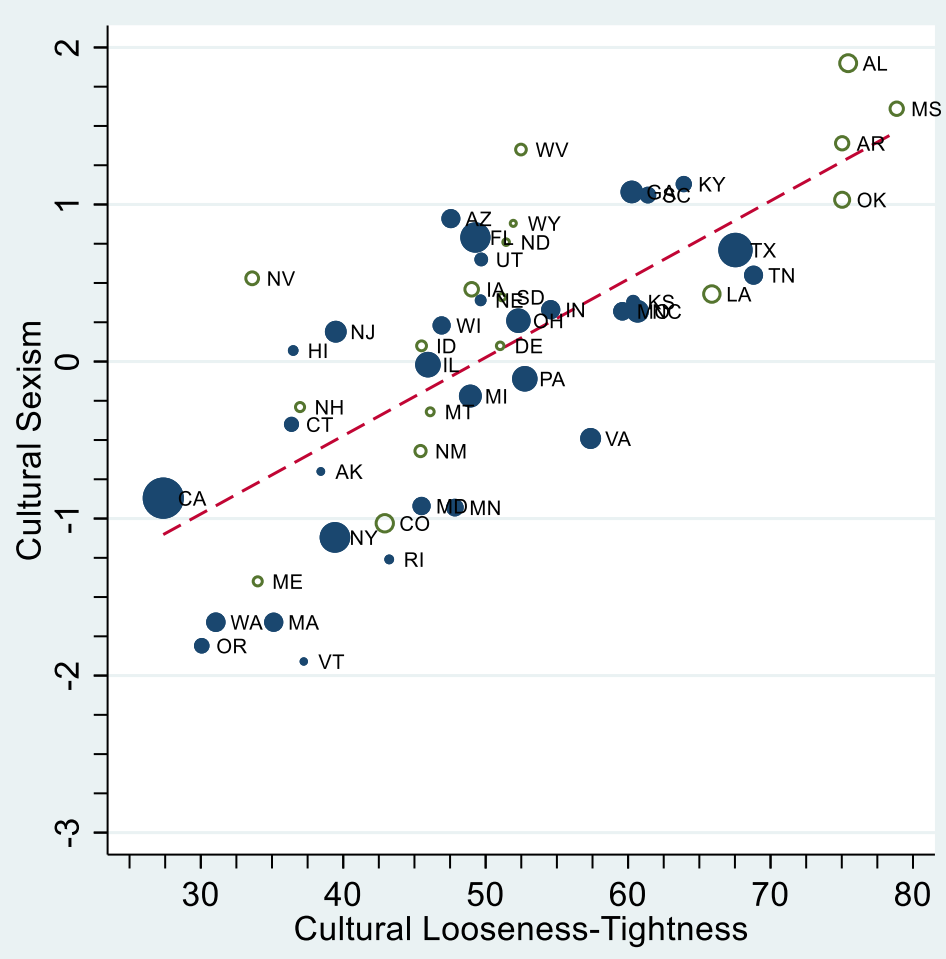

Command:

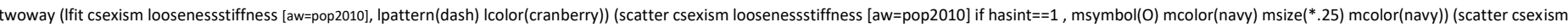
loosenessstiffness [aw=pop2010] if hasint $==0$, mcolor(forest_green) msymbol(Oh) msize( $\left.{ }^{*} .25\right)$ ) (scatter csexism loosenessstiffness, symbol(i) mlabel(postalcode) mlabcolor(black) mlabsize(vsmall)), aspect(1) xtick(25(2.5)80) ytick(-3(.25)2) ytitle(Cultural Sexism) xtitle(Cultural Looseness-Tightness) legend(off)

DC's Looseness-Tightness can be interpolated from its cultural sexism and slaveholding scores:

- reg loosenessstiffness pctslaves1860 csexism, b

\begin{tabular}{|c|c|c|c|c|c|c|}
\hline Source & SS & $d f$ & MS & Number of obs & $=$ & 50 \\
\hline Model & 5301.04208 & 2 & 2650.52104 & Prob > F & $=$ & 0.0000 \\
\hline Residual & 2473.05114 & 47 & 52.6181094 & R-squared & $=$ & 0.6819 \\
\hline Total & 7774.09323 & 49 & 158.654964 & Root MSE & $=$ & 7.2538 \\
\hline
\end{tabular}

\begin{tabular}{r|rrrrr}
\hline loosenessst s & Coef. & Std. Err. & $t$ & $\mathrm{P}>|\mathrm{t}|$ & Beta \\
\hline pctslaves1860 & .2695371 & .0690593 & 3.90 & 0.000 & .3701247 \\
csexism & 7.813829 & 1.284909 & 6.08 & 0.000 & .5766907 \\
_cons & 47.13894 & 1.205116 & 39.12 & 0.000 &. \\
\hline
\end{tabular}

- predict 1s_sl_sexism

(option $\mathbf{x b}$ assumed; fitted values)

- lis loosenessstiffness csexism 1s_hat 1s_sl_sexism if postalcode=="DC"

9.

\begin{tabular}{|crrr|}
\hline loosen s & csexism & ls_hat & ls_sl_ m \\
\hline$\cdot$ & -2.61 & 16.97957 & 27.93081 \\
\hline
\end{tabular}

The Is_hat is the predicted value from the regression with just csexism (not shown). Use the Is_sl_sexism estimate as more reasonable and less extreme, now in the variable $1 \mathrm{~s}$. 


\section{Technical Summary}

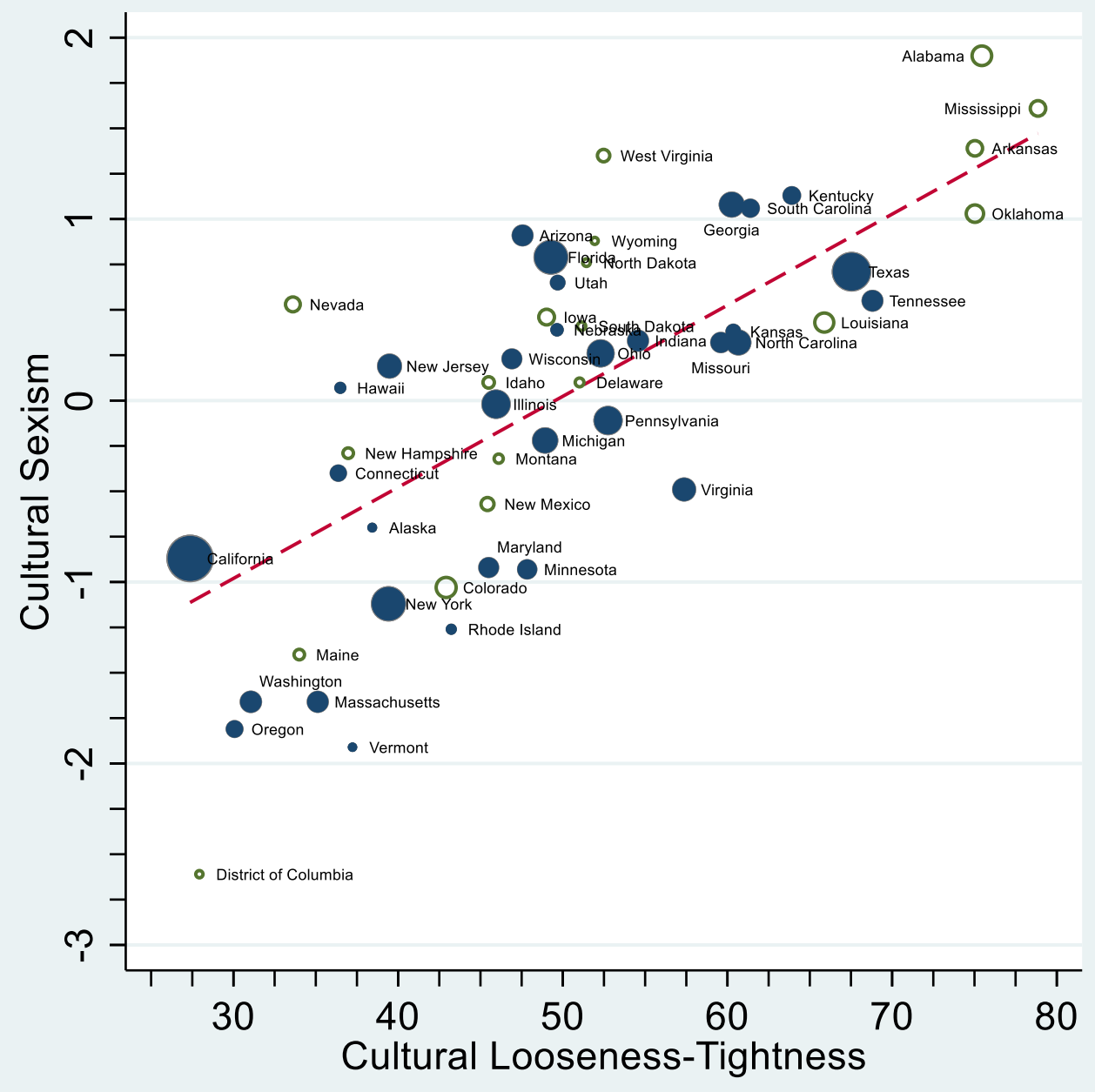

Command:

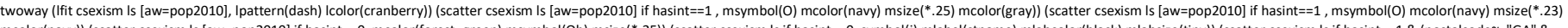

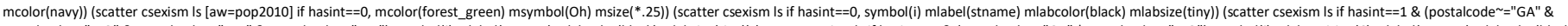

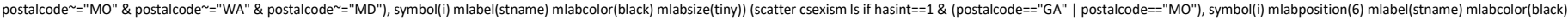

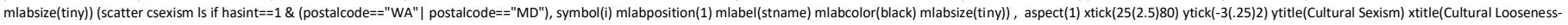
Tightness) legend(off) 


\section{Technical Summary}

If the population weights are removed, a quadratic function can be seen:

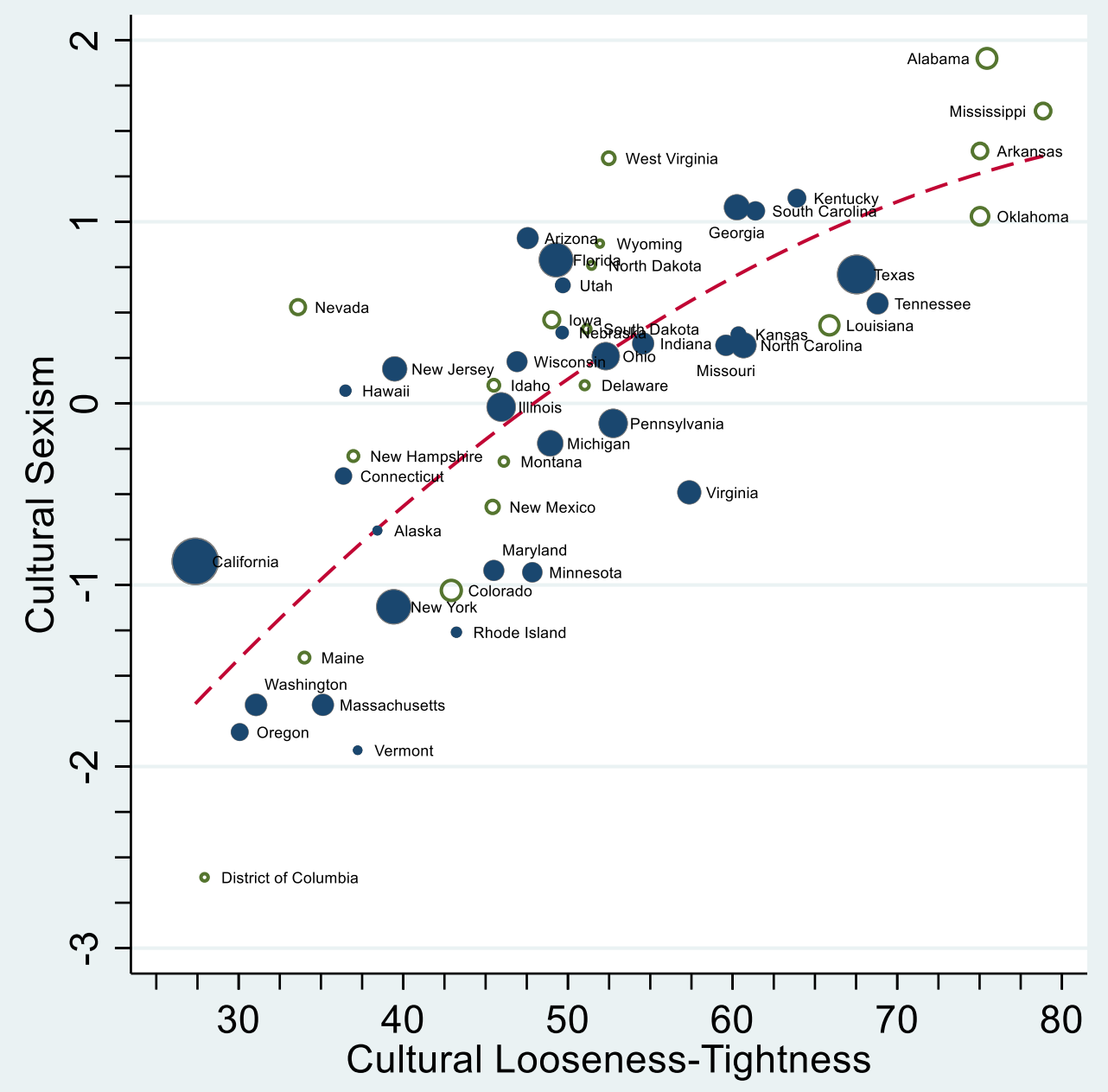

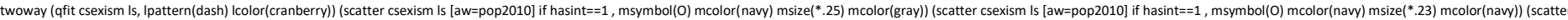

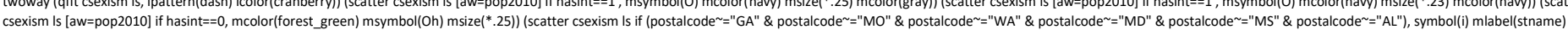

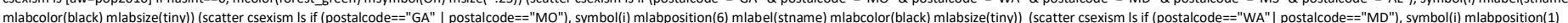

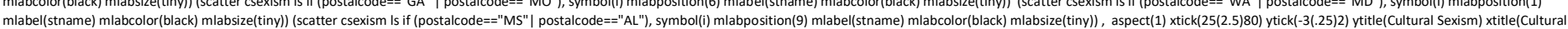
mabel(stnam) mabcolor 


\section{Technical Summary}

\section{State-Level Sex Education}

Guttmacher sex education items:

- sum cse-sexorientation

\begin{tabular}{|c|c|c|c|c|c|}
\hline Variable & Obs & Mean & Std. Dev. & Min & $\operatorname{Max}$ \\
\hline cse & 47 & .6382979 & .4856879 & 0 & 1 \\
\hline hiv & 47 & .8297872 & .3798826 & 0 & 1 \\
\hline medacc & 47 & .4255319 & .4997687 & 0 & 1 \\
\hline contracept n & 44 & .4772727 & .5052578 & 0 & 1 \\
\hline condoms & 44 & .4545455 & .5036862 & 0 & 1 \\
\hline abstinence & 44 & -.6136364 & .5376914 & -1 & 1 \\
\hline sexinmarri e & 44 & -.4318182 & .501056 & -1 & 0 \\
\hline sexorienta n & 44 & .1363636 & .6321212 & -1 & 1 \\
\hline
\end{tabular}

- pwcorr cse-sexorientation

\begin{tabular}{|c|c|c|c|c|c|c|c|}
\hline & cse & hiv & medacc & contra n & condoms & abstin e & sexinm e \\
\hline cse & 1.0000 & & & & & & \\
\hline hiv & 0.3660 & 1.0000 & & & & & \\
\hline medacc & -0.0686 & -0.0682 & 1.0000 & & & & \\
\hline contracept n & 0.3671 & 0.2592 & 0.2082 & 1.0000 & & & \\
\hline condoms & 0.3320 & 0.2410 & 0.3457 & 0.8640 & 1.0000 & & \\
\hline abstinence & 0.1776 & 0.1563 & -0.2151 & 0.1615 & 0.1093 & 1.0000 & \\
\hline sexinmarri e & 0.1870 & 0.0471 & -0.0153 & 0.2818 & 0.3351 & 0.4610 & 1.0000 \\
\hline sexorienta n & 0.2462 & -0.0173 & 0.0892 & 0.3012 & 0.2390 & 0.3888 & 0.5574 \\
\hline
\end{tabular}

They make a reasonable scale:

- alpha cse-sexorientation

Test scale $=$ mean (unstandardized items)

Average interitem covariance: $\quad .0593777$

Number of items in the scale: 8

Scale reliability coefficient: $\quad 0.7045$ 


\section{Technical Summary}

And a simple factor analysis suggests one factor.

- factor cse-sexorientation

(obs=43)

Factor analysis/correlation Method: principal factors

Number of obs $=43$

Rotation: (unrotated)

Retained factors $=\quad 4$

Number of params $=\quad 26$

\begin{tabular}{c|rrrr}
\hline Factor & Eigenvalue & Difference & Proportion & Cumulative \\
\hline Factor1 & 2.44439 & 1.45871 & 0.6895 & 0.6895 \\
Factor2 & 0.98568 & 0.35411 & 0.2780 & 0.9675 \\
Factor3 & 0.63157 & 0.55074 & 0.1782 & 1.1457 \\
Factor4 & 0.08083 & 0.11204 & 0.0228 & 1.1685 \\
Factor5 & -0.03121 & 0.10153 & -0.0088 & 1.1597 \\
Factor6 & -0.13274 & 0.05794 & -0.0374 & 1.1223 \\
Factor7 & -0.19068 & 0.05205 & -0.0538 & 1.0685 \\
Factor8 & -0.24272 &. & -0.0685 & 1.0000 \\
\hline
\end{tabular}

LR test: independent vs. saturated: $\operatorname{chi2}(28)=117.29$ Prob $>$ chi2 $=0.0000$

Factor loadings (pattern matrix) and unique variances

\begin{tabular}{r|rrrr|r}
\hline Variable & Factor1 & Factor2 & Factor3 & Factor4 & Uniqueness \\
\hline cse & 0.4530 & 0.0530 & 0.3260 & 0.1726 & 0.6559 \\
hiv & 0.2908 & -0.0022 & 0.4928 & 0.0493 & 0.6702 \\
medacc & 0.1603 & -0.4010 & -0.3916 & 0.1241 & 0.6447 \\
contracept n & 0.8347 & -0.3075 & 0.0581 & -0.0839 & 0.1982 \\
condoms & 0.8349 & -0.3981 & -0.0428 & -0.0606 & 0.1390 \\
abstinence & 0.3860 & 0.4827 & 0.0559 & -0.0868 & 0.6073 \\
sexinmarri e & 0.5642 & 0.4201 & -0.2152 & -0.0339 & 0.4578 \\
sexorienta n & 0.5175 & 0.3994 & -0.2729 & 0.1176 & 0.4844 \\
\hline
\end{tabular}

If we make the scale the mean of available items, then:

. sum mnsexed

Variable | Obs Mean Std. Dev. Min Max

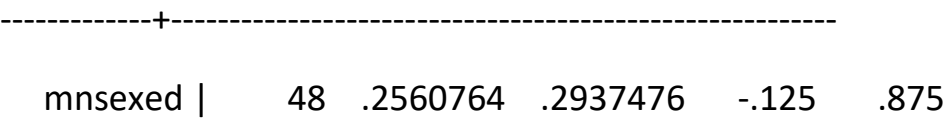

Of note, there are 3 that have no information. These do not differ on average on either sexism or tightness (see two analyses following). 


\section{Technical Summary}

- anova csexism nosexedinfo

\begin{tabular}{r|rrrrrr}
\multicolumn{2}{c}{$\begin{array}{l}\text { Number of obs } \\
\text { Root MSE }\end{array}$} & $\begin{array}{r}51 \\
1.00185\end{array}$ & $\begin{array}{l}\text { R-squared } \\
\text { Adj R-squared }=\end{array}$ & -0.0180 \\
Source & Partial SS & df & MS & F & Prob $>F$ \\
\hline Model & .11459423 & & 1 & .11459423 & 0.11 & 0.7369 \\
nosexedinfo & .11459423 & & 1 & .11459423 & 0.11 & 0.7369 \\
Residual & 49.181397 & 49 & 1.003702 & & \\
\hline Total & 49.295991 & 50 & .98591982 & &
\end{tabular}

- anova ls nosexedinfo

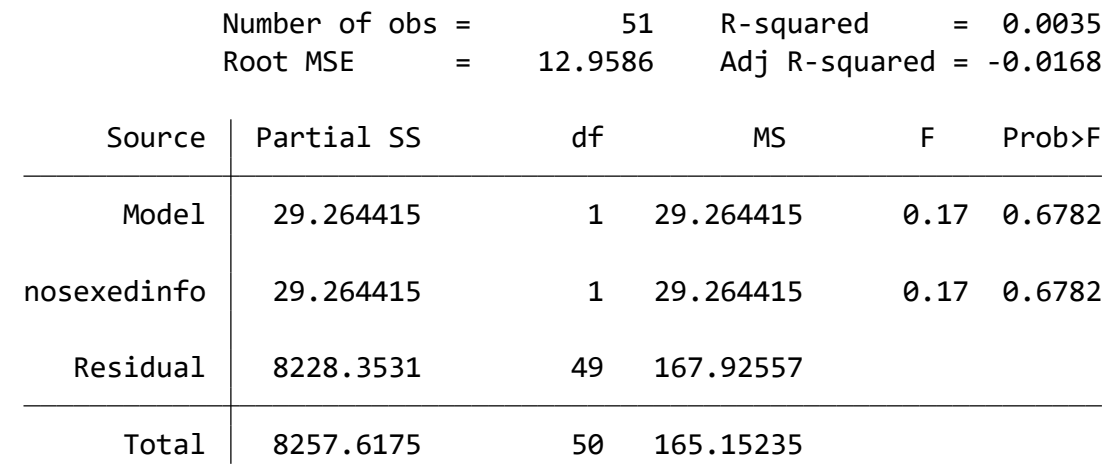

Sex education correctness is significantly correlated with the focal variables in question:

. pwcorr mnsexed csexism is pctslaves1860 hasint pop2010, $\operatorname{star}(.05)$

\begin{tabular}{r|cccccc} 
& mnsexed & csexism & ls pct 1860 & hasint pop2010 \\
\hline mnsexed & 1.0000 & & & & & \\
csexism & $-0.5544^{*}$ & 1.0000 & & & & \\
1 ls & $-0.6085^{*}$ & $0.7750^{*}$ & 1.0000 & & & \\
pctslav 1860 & $-0.4109^{*}$ & $0.4765^{*}$ & $0.6471^{*}$ & 1.0000 & & \\
hasint & -0.0702 & -0.1948 & -0.1447 & 0.0044 & 1.0000 & \\
pop2010 & 0.0061 & -0.0190 & -0.0404 & 0.1623 & $0.4360^{*}$ & 1.0000
\end{tabular}

It doesn't change much if you weight for pop:

- pwcorr mnsexed csexism ls pctslaves1860 hasint pop2010 [aw=pop2010], star(.05)

\begin{tabular}{r|cccccc} 
& mnsexed & csexism & ls pct 1860 & hasint pop2010 \\
\hline mnsexed & 1.0000 & & & & & \\
csexism & $-0.5493^{*}$ & 1.0000 & & & & \\
$1 s$ & $-0.6273^{*}$ & $0.7868^{*}$ & 1.0000 & & & \\
pctslav 1860 & $-0.3842^{*}$ & $0.6203^{*}$ & $0.6418^{*}$ & 1.0000 & & \\
hasint & 0.0352 & -0.2285 & -0.2333 & -0.0718 & 1.0000 & \\
pop2010 & $0.4941^{*}$ & -0.2478 & $-0.4031^{*}$ & -0.0488 & $0.3801^{*}$ & 1.0000
\end{tabular}




\section{Technical Summary}

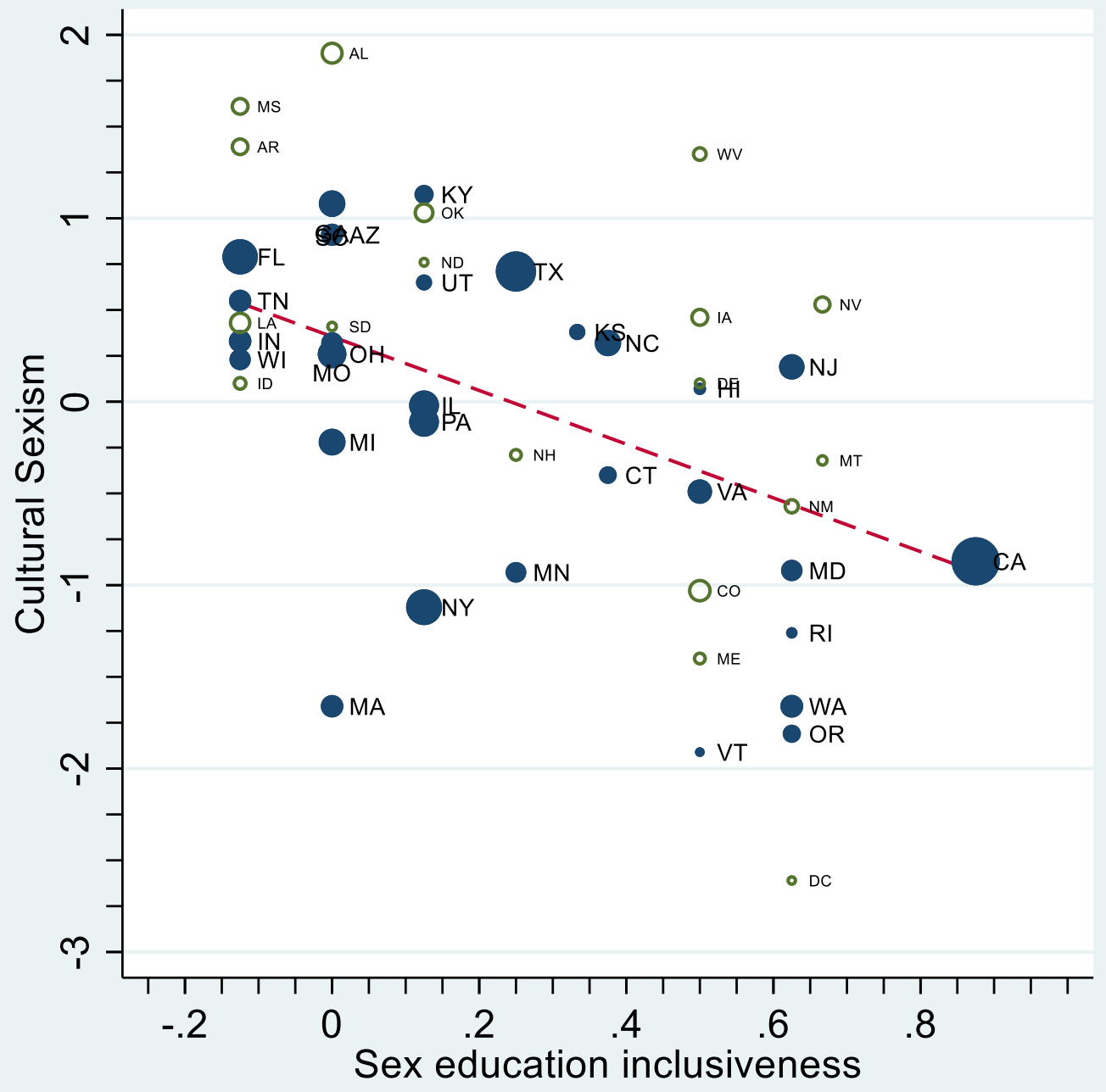

Command:

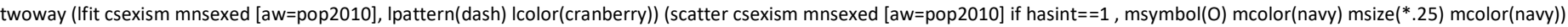

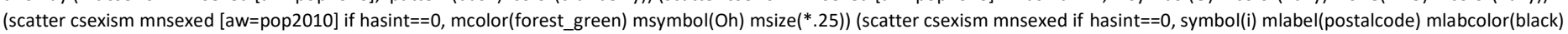

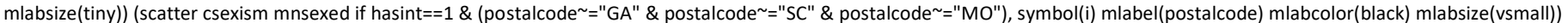

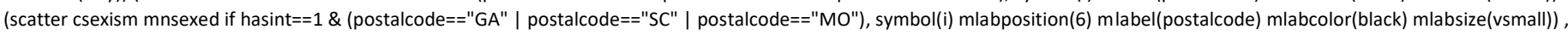
aspect(1) xtick(-.25(.05)1) ytick(-3(.25)2) ytitle(Cultural Sexism) xtitle(Sex education inclusiveness) legend(off)

\section{Or rotated:}




\section{Technical Summary}

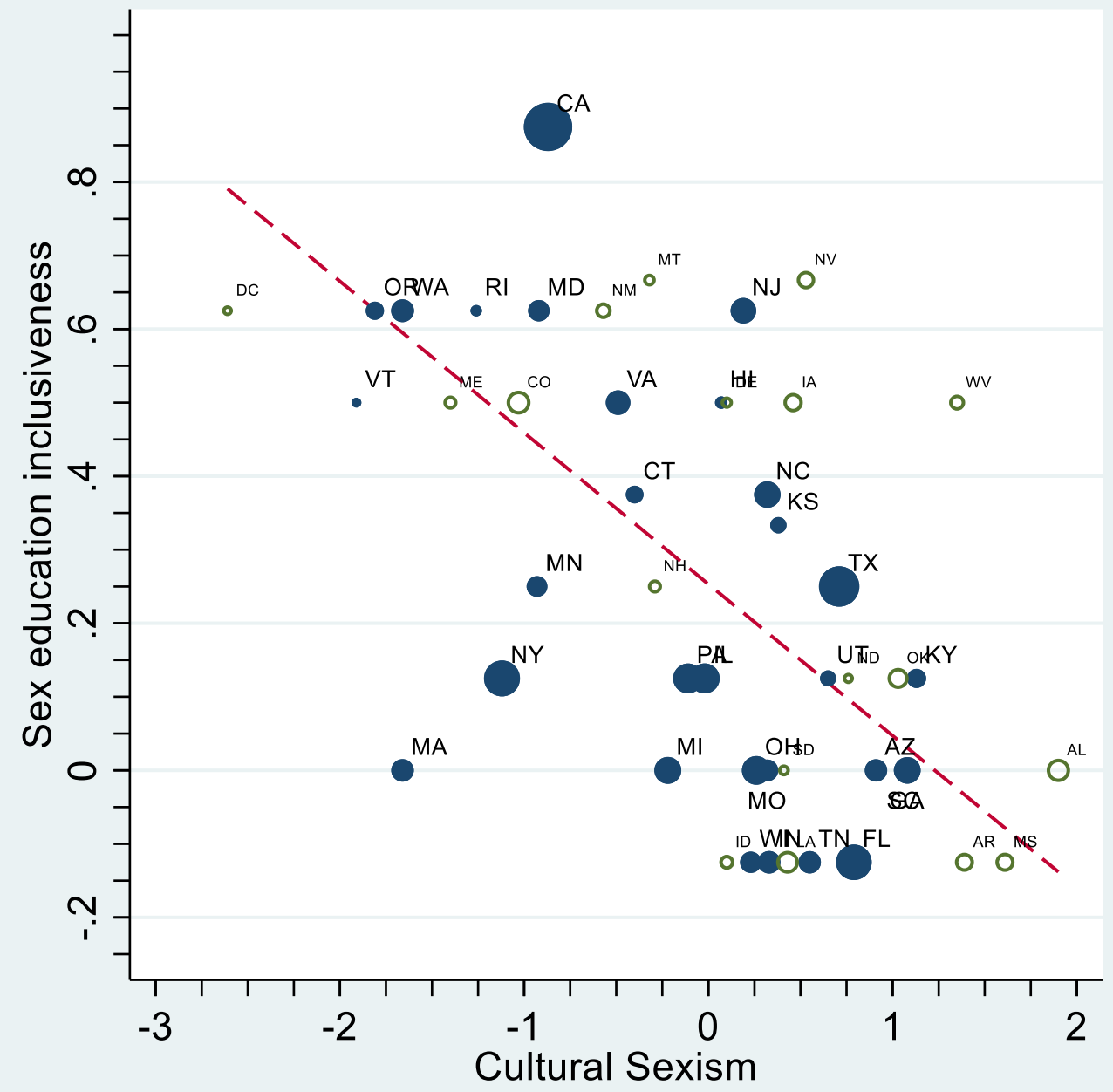

Command:

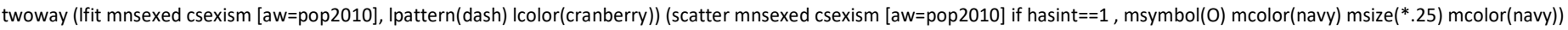

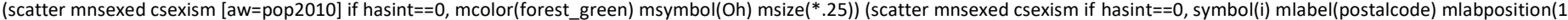
mlabcolor(black) mlabsize(tiny)) (scatter mnsexed csexism if hasint==1 \& (postalcode $=" G A " ~ \&$ postalcode $=" S C$ " \& postalcode $=" M O ")$, symbol(i) mlabel(postalcode) mlabposition(1)

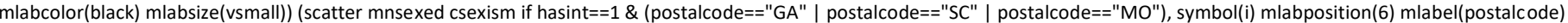
mlabcolor(black) mlabsize(vsmall)), aspect(1) ytick(-.25(.05)1) xtick(-3(.25)2) xtitle(Cultural Sexism) ytitle(Sex education inclusiveness) legend(off) 


\section{Technical Summary}

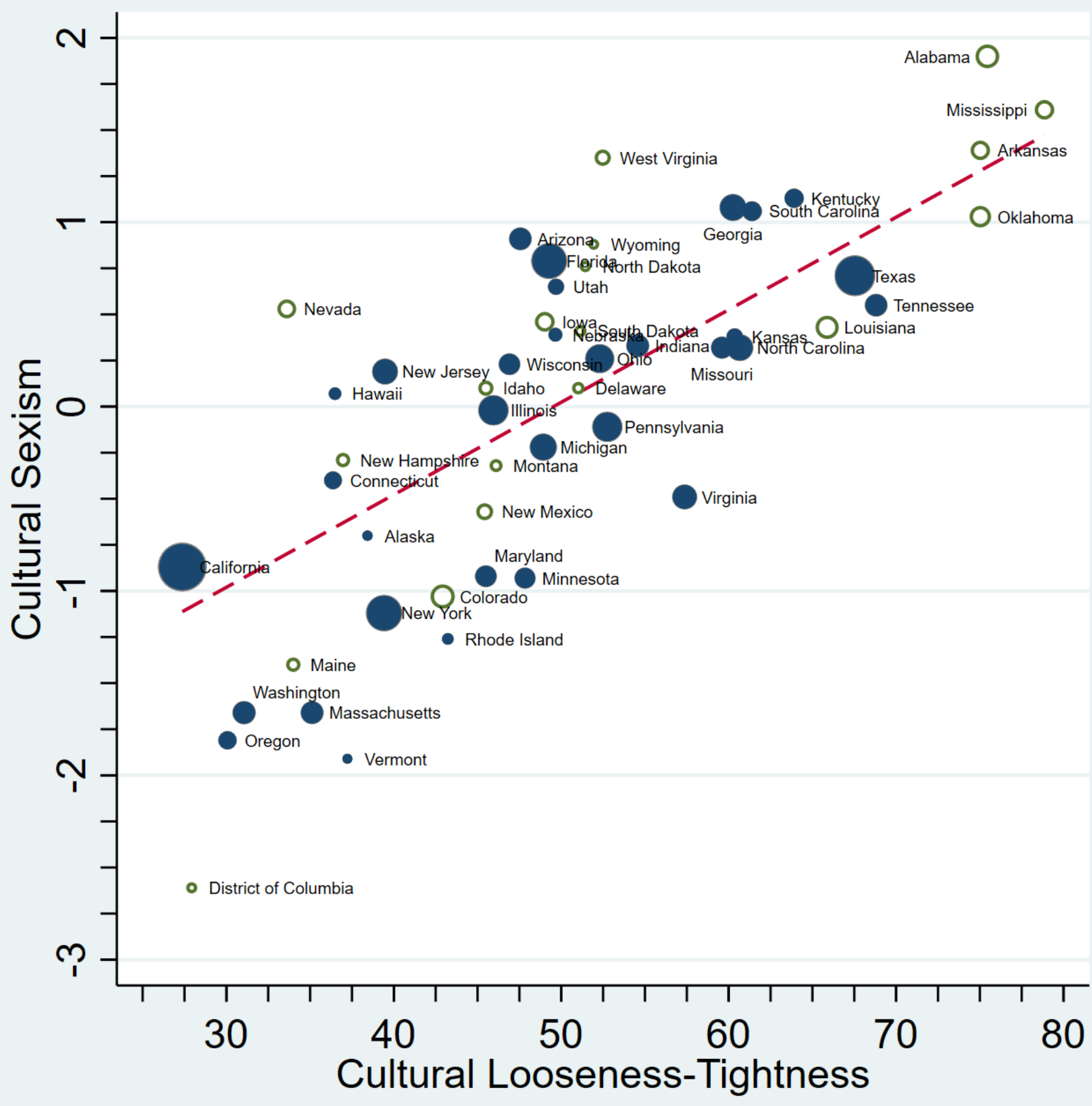

Command:

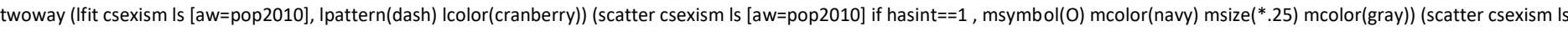

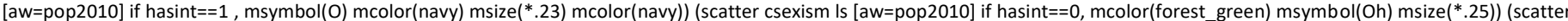

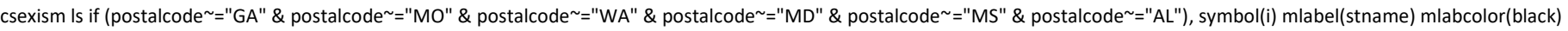
mlabsize(tiny)) (scatter csexism Is if (postalcode=="GA" | postalcode=="MO"), symbol(i) mlabposition(6) mlabel(stname) mlabcolor(black) mlabsize(tiny)) (scatter csexism Is if

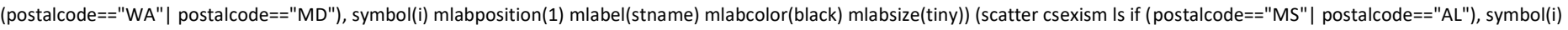
mlabposition(9) mlabel(stname) mlabcolor(black) mlabsize(tiny)), aspect(1) xtick(25(2.5)80) ytick(-3(.25)2) ytitle(Cultural Sexism) xtitle(Cultural Looseness-Tightness) legend(off) 


\section{Technical Summary}

Inclusiveness is related to sexism whether without (first) or with population weighting (second):

- reg mnsexed csexism, b

\begin{tabular}{|c|c|c|c|c|c|c|}
\hline Source & SS & $d f$ & MS & Number of obs & $=$ & 48 \\
\hline Model & 1.24653238 & 1 & 1.24653238 & Prob > F & $=$ & 0.0000 \\
\hline Residual & 2.80898704 & 46 & .061064936 & R-squared & $=$ & 0.3074 \\
\hline & & & & Adj R-squared & $=$ & 0.2923 \\
\hline Total & 4.05551942 & 47 & .086287647 & Root MSE & $=$ & .24711 \\
\hline
\end{tabular}

\begin{tabular}{r|rrrrr}
\hline mnsexed & Coef. & Std. Err. & $t$ & $P>|t|$ & Beta \\
\hline csexism & -.1613636 & .0357149 & -4.52 & 0.000 & -.5544068 \\
_cons & .2542274 & .0356701 & 7.13 & 0.000 &. \\
\hline
\end{tabular}

- reg mnsexed csexism [aw=pop2010], b (sum of wgt is $305,645,340$ )

\begin{tabular}{|c|c|c|c|c|c|c|}
\hline Source & SS & $d f$ & MS & Number of obs & $=$ & 48 \\
\hline Model & 1.53605535 & 1 & 1.53605535 & Prob $>\mathrm{F}$ & $=$ & 0.0001 \\
\hline Residual & 3.55499522 & 46 & .077282505 & R-squared & $=$ & 0.3017 \\
\hline Total & 5.09105057 & 47 & .108320225 & Root MSE & $=$ & .278 \\
\hline
\end{tabular}

\begin{tabular}{r|rrrrr}
\hline mnsexed & Coef. & Std. Err. & $t$ & $P>|t|$ & Beta \\
\hline csexism & -.2059436 & .046194 & -4.46 & 0.000 & -.5492875 \\
_cons & .253255 & .0401394 & 6.31 & 0.000 &. \\
\hline
\end{tabular}

\section{Lifetime Intimate Sexual Violence}

We used CDC data from the 2010-2012 State Report to document lifetime intimate partner violence data by gender, which was available for 49 of 51 states. An odds ratio was defined to document this tendency, which across states generally meant that females experienced more than did males.

. sum oripvgender

\begin{tabular}{r|rrrrr} 
Variable & Obs & Mean & Std. Dev. & Min & Max \\
\hline oripvgender & 49 & 1.453032 & .5007297 & .7221897 & 3.36762
\end{tabular}




\section{Technical Summary}

Those missing data were two states low in cultural sexism.

- anova csexism noipv

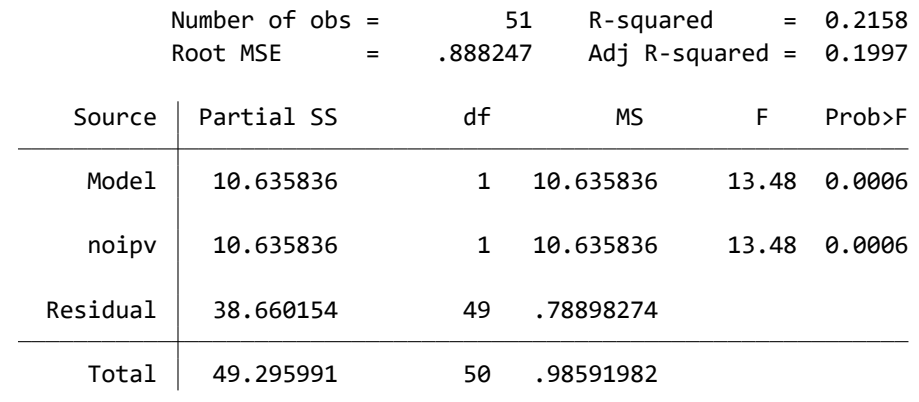

- tab noipv, sum(csexism)

\begin{tabular}{r|rcr}
\multirow{2}{*}{ noipv } & \multicolumn{3}{|c}{ Summary of csexism } \\
& Mean & Std. Dev. & Freq. \\
\hline 0 & .09265306 & .89460366 & 49 \\
1 & -2.2599999 & .4949747 & 2 \\
\hline Total & .00039216 & .99293495 & 51
\end{tabular}

. lis state if noipv $==1$

9.

\begin{tabular}{|r|}
\hline state \\
\hline DISTRICT OF COLUMBIA \\
VERMONT \\
\hline
\end{tabular}

These two were also relatively loose states, although not markedly different:

- anova ls noipv

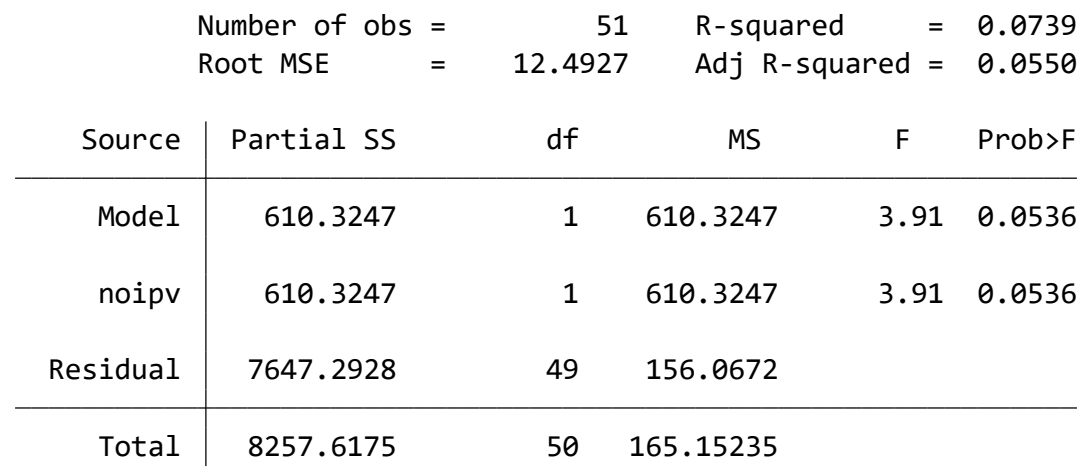

- tab noipv, sum(ls)

\begin{tabular}{r|rrr}
\multirow{2}{*}{ noipv } & \multicolumn{3}{|c}{ Summary of ls } \\
& Mean & Std. Dev. & Freq. \\
\hline 0 & 50.402244 & 12.586414 & 49 \\
1 & 32.580405 & 6.5755193 & 2 \\
\hline Total & 49.703349 & 12.851161 & 51
\end{tabular}




\section{Technical Summary}

- pwcorr oripvgender csexism is pctslaves1860 mnsexed hasint pop2010 [aw=pop2010], star(.05)

\begin{tabular}{|c|c|c|c|c|c|c|c|}
\hline & oripvg r & csexism & Is & pct 1860 & mnsexed & hasint & pop2010 \\
\hline oripvgender & 1.0000 & & & & & & \\
\hline csexism & 0.1538 & 1.0000 & & & & & \\
\hline ls & 0.1405 & $0.7868 *$ & 1.0000 & & & & \\
\hline pctslav 1860 & 0.2052 & $0.6203 *$ & $0.6418^{*}$ & 1.0000 & & & \\
\hline mnsexed & -0.1227 & $-0.5493 *$ & $-0.6273 *$ & $-0.3842 *$ & 1.0000 & & \\
\hline hasint & 0.1686 & -0.2285 & -0.2333 & -0.0718 & 0.0352 & 1.0000 & \\
\hline pop2010 & $-0.3047 *$ & -0.2478 & $-0.4031 *$ & -0.0488 & $0.4941 *$ & $0.3801 *$ & 1.0000 \\
\hline
\end{tabular}

Intimate partner violence was uncorrelated with the primary variables of this study.

Mean levels reporting lifetime sexual violence were also not correlated.

- sum lifesexvio

\begin{tabular}{r|rrrrr} 
Variable & Obs & Mean & Std. Dev. & Min & Max \\
\hline lifesexvio & 51 & 37.05098 & 3.909341 & 29.5 & 47.5
\end{tabular}

- pwcorr lifesexvio oripvgender csexism ls pctslaves1860 mnsexed hasint pop2010 [aw=pop2010], star(.05)

\begin{tabular}{r|rrrrrrr} 
& lifese 0 oripvg r & csexism & ls pct 1860 & mnsexed & hasint \\
\hline lifesexvio & 1.0000 & & & & & & \\
oripvgender & -0.0039 & 1.0000 & & & & & \\
csexism & -0.2496 & 0.1538 & 1.0000 & & & & \\
ls & -0.1366 & 0.1405 & $0.7868^{*}$ & 1.0000 & & & \\
pctslav 1860 & $-0.3375^{*}$ & 0.2052 & $0.6203^{*}$ & $0.6418^{*}$ & 1.0000 & & \\
mnsexed & 0.0972 & -0.1227 & $-0.5493^{*}$ & $-0.6273^{*}$ & $-0.3842^{*}$ & 1.0000 & \\
hasint & 0.1411 & 0.1686 & -0.2285 & -0.2333 & -0.0718 & 0.0352 & 1.0000 \\
pop2010 & -0.2450 & $-0.3047^{*}$ & -0.2478 & $-0.4031^{*}$ & -0.0488 & $0.4941^{*}$ & $0.3801^{*}$
\end{tabular}


Technical Summary

Variables State by State

\begin{tabular}{|c|c|c|c|c|c|c|c|c|c|c|}
\hline & stname & postal e & stfips & csexism & hasint & Is_rank & loosen s & pop2010 & ginirank & gini \\
\hline 1. & Alabama & $\mathrm{AL}$ & 1 & 1.9 & 0 & 2 & 75.45 & $4.8 \mathrm{e}+06$ & 45 & .4847 \\
\hline 2. & Alaska & AK & 2 & -.7 & 1 & 40 & 38.43 & 710231 & 2 & .4081 \\
\hline 3. & Arizona & $A Z$ & 4 & .91 & 1 & 29 & 47.56 & $6.4 \mathrm{e}+06$ & 31 & .4713 \\
\hline 4. & Arkansas & $A R$ & 5 & 1.39 & $\theta$ & 3 & 75.03 & $2.9 e+06$ & 32 & .4719 \\
\hline 5. & California & CA & 6 & -.87 & 1 & 50 & 27.37 & $3.7 \mathrm{e}+07$ & 48 & .4899 \\
\hline 6. & Colorado & co & 8 & -1.03 & $\theta$ & 37 & 42.92 & $5.0 e+06$ & 21 & .4586 \\
\hline 7. & Connecticut & СT & 9 & -.4 & 1 & 44 & 36.37 & $3.6 \mathrm{e}+06$ & 49 & .4945 \\
\hline 8. & Delaware & $\mathrm{DE}$ & 10 & $\begin{array}{llll}.1 & & & \\
\end{array}$ & $\theta$ & 22 & 51.02 & 897934 & 14 & .4522 \\
\hline 9. & District of Columbia & DC & 11 & -2.61 & $\theta$ & & & 601723 & 52 & .542 \\
\hline 10. & Florida & $\mathrm{FL}$ & 12 & .79 & 1 & 25 & 49.28 & $1.9 \mathrm{e}+07$ & 46 & .4852 \\
\hline 11. & Georgia & GA & 13 & 1.08 & 1 & 12 & 60.26 & $9.7 \mathrm{e}+06$ & 43 & .4813 \\
\hline 12. & Hawaii & $\mathrm{HI}$ & 15 & .07 & 1 & 43 & 36.49 & $1.4 \mathrm{e}+06$ & 5 & .442 \\
\hline 13. & Idaho & ID & 16 & .1 & $\theta$ & 33 & 45.5 & $1.6 \mathrm{e}+06$ & 12 & .4503 \\
\hline 14. & Illinois & IL & 17 & -.02 & 1 & 32 & 45.95 & $1.3 e+07$ & 40 & .481 \\
\hline 15. & Indiana & IN & 18 & .33 & 1 & 15 & 54.57 & $6.5 e+06$ & 15 & .4527 \\
\hline 16. & Iowa & IA & 19 & .46 & $\theta$ & 26 & 49.02 & $3.0 \mathrm{e}+06$ & 6 & .4451 \\
\hline 17. & Kansas & KS & 20 & .38 & 1 & 11 & 60.36 & $2.9 e+06$ & 18 & .455 \\
\hline 18. & Kentucky & KY & 21 & 1.13 & 1 & 8 & 63.91 & $4.3 e+06$ & 42 & .4813 \\
\hline 19. & Louisiana & LA & 22 & .43 & $\theta$ & 7 & 65.88 & $4.5 e+06$ & 50 & .499 \\
\hline 20. & Maine & ME & 23 & -1.4 & $\theta$ & 46 & 34 & $1.3 e+06$ & 13 & .4519 \\
\hline 21. & Maryland & MD & 24 & -.92 & 1 & 34 & 45.5 & $5.8 \mathrm{e}+06$ & 11 & .4499 \\
\hline 22. & Massachusetts & MA & 25 & -1.66 & 1 & 45 & 35.12 & $6.5 e+06$ & 37 & .4786 \\
\hline 23. & Michigan & MI & 26 & -.22 & 1 & 27 & 48.93 & $9.9 e+06$ & 28 & .4695 \\
\hline 24. & Minnesota & MN & 27 & -.93 & 1 & 28 & 47.84 & $5.3 e+06$ & 9 & .4496 \\
\hline 25. & Mississippi & MS & 28 & 1.61 & $\theta$ & 1 & 78.86 & $3.0 \mathrm{e}+06$ & 44 & .4828 \\
\hline 26. & Missouri & MO & 29 & .32 & 1 & 13 & 59.6 & $6.0 \mathrm{e}+06$ & 24 & .4646 \\
\hline 27. & Montana & MT & 30 & -.32 & 0 & 31 & 46.11 & 989415 & 25 & .4667 \\
\hline 28. & Nebraska & $\mathrm{NE}$ & 31 & .39 & 1 & 24 & 49.65 & $1.8 \mathrm{e}+06$ & 7 & .4477 \\
\hline 29. & Nevada & NV & 32 & .53 & 0 & 47 & 33.61 & $2.7 e+06$ & 19 & .4577 \\
\hline 30. & New Hampshire & $\mathrm{NH}$ & 33 & -.29 & $\theta$ & 42 & 36.97 & $1.3 e+06$ & 3 & .4304 \\
\hline 31. & New Jersey & $\mathrm{NJ}$ & 34 & .19 & 1 & 38 & 39.48 & $8.8 \mathrm{e}+06$ & 41 & .4813 \\
\hline 32. & New Mexico & NM & 35 & -.57 & $\theta$ & 35 & 45.43 & $2.1 \mathrm{e}+06$ & 34 & .4769 \\
\hline 33. & New York & NY & 36 & -1.12 & 1 & 39 & 39.42 & $1.9 e+07$ & 51 & .5229 \\
\hline 34. & North Carolina & $\mathrm{NC}$ & 37 & .32 & 1 & 10 & 60.67 & $9.5 e+06$ & 35 & .478 \\
\hline 35. & North Dakota & ND & 38 & .76 & $\theta$ & 20 & 51.44 & 672591 & 16 & .4533 \\
\hline 36. & Ohio & $\mathrm{OH}$ & 39 & .26 & 1 & 18 & 52.3 & $1.2 \mathrm{e}+07$ & 26 & .468 \\
\hline 37. & Oklahoma & $\mathrm{OK}$ & 40 & 1.03 & $\theta$ & 4 & 75.03 & $3.8 \mathrm{e}+06$ & 23 & .4645 \\
\hline 38. & Oregon & OR & 41 & -1.81 & 1 & 49 & 30.07 & $3.8 \mathrm{e}+06$ & 20 & .4583 \\
\hline 39. & Pennsylvania & PA & 42 & -.11 & 1 & 16 & 52.75 & $1.3 e+07$ & 27 & .4689 \\
\hline 40. & Rhode Island & RI & 44 & -1.26 & 1 & 36 & 43.23 & $1.1 \mathrm{e}+06$ & 36 & .4781 \\
\hline 41. & South Carolina & SC & 45 & 1.06 & 1 & 9 & 61.39 & $4.6 \mathrm{e}+06$ & 33 & .4735 \\
\hline 42. & South Dakota & SD & 46 & .41 & 0 & 21 & 51.14 & 814180 & 8 & .4495 \\
\hline 43. & Tennessee & TN & 47 & .55 & 1 & 5 & 68.81 & $6.3 e+06$ & 38 & .479 \\
\hline 44. & Texas & TX & 48 & .71 & 1 & 6 & 67.54 & $2.5 e+07$ & 39 & .48 \\
\hline 45. & Utah & UT & 49 & .65 & 1 & 23 & 49.69 & $2.8 \mathrm{e}+06$ & 1 & .4063 \\
\hline 46. & Vermont & VT & 50 & -1.91 & 1 & 41 & 37.23 & 625741 & 17 & .4539 \\
\hline 47. & Virginia & VA & 51 & -.49 & 1 & 14 & 57.37 & $8.0 e+06$ & 29 & .4705 \\
\hline 48. & Washington & WA & 53 & -1.66 & 1 & 48 & 31.06 & $6.7 e+06$ & 22 & .4591 \\
\hline 49. & West Virginia & wV & 54 & 1.35 & $\theta$ & 17 & 52.48 & $1.9 e+06$ & 30 & .4711 \\
\hline 50. & Wisconsin & WI & 55 & .23 & 1 & 30 & 46.91 & $5.7 e+06$ & 10 & .4498 \\
\hline 51. & Wyoming & WY & 56 & .88 & $\theta$ & 19 & 51.94 & 563626 & 4 & .436 \\
\hline
\end{tabular}


Technical Summary

. lis csexloosehat - 1s_sl_sexism, clean

\begin{tabular}{|c|c|c|c|c|c|c|}
\hline & csexloo t & csexloo s & 1s_hat & ls & lcislr & ucislr \\
\hline 1. & 1.294576 & .6054239 & 72.93412 & 75.45 & .4567568 & .754091 \\
\hline 2. & -.5507428 & -1492572 & 40.67651 & 38.43 & -.2979243 & -.00059 \\
\hline 3. & -.0956438 & 1.005644 & 60.65142 & 47.56 & .8569767 & 1.154311 \\
\hline 4. & 1.273641 & .1163594 & 66.60667 & 75.03 & -.0323077 & .2650265 \\
\hline 5. & -1.102046 & .2320456 & 38.56736 & 27.37 & .0833785 & .3807127 \\
\hline 6. & -.326932 & -.703068 & 36.58228 & 42.92 & -.8517351 & -.5544009 \\
\hline 7. & -.6534268 & .2534268 & 44.39854 & 36.37 & .1047597 & .4020939 \\
\hline 8. & .0768252 & .0231748 & 50.60193 & 51.02 & -.1254923 & .1718419 \\
\hline 9. & & & 16.97957 & 27.93081 & & \\
\hline 10. & -.0099079 & .7999079 & 59.1626 & 49.28 & .6512408 & .948575 \\
\hline 11. & .5374072 & .5425929 & 62.76057 & 60.26 & .3939258 & .69126 \\
\hline 12. & -.6474451 & .7174451 & 50.22972 & 36.49 & .568778 & .8661122 \\
\hline 13. & -.1983278 & .2983278 & 50.60193 & 45.5 & .1496607 & .4469949 \\
\hline 14. & -.1758968 & .1558968 & 49.11311 & 45.95 & .0072297 & .3045639 \\
\hline 15. & .2537804 & .0762196 & 53.45549 & 54.57 & -.0724475 & .2248868 \\
\hline 16. & -.0228679 & .4828679 & 55.06837 & 49.02 & .3342008 & .6315351 \\
\hline 17. & .5423919 & -1623919 & 54.07582 & 60.36 & -.311059 & -.0137248 \\
\hline 18. & .7193471 & .4106529 & 63.38091 & 63.91 & .2619858 & .55932 \\
\hline 19. & .8175446 & -.3875447 & 54.69616 & 65.88 & -.5362118 & -.2388775 \\
\hline 20. & -.7715631 & -.6284369 & 31.99177 & 34 & -.777104 & -.4797698 \\
\hline 21. & -1983278 & -.7216722 & 37.94702 & 45.5 & -.8703393 & -.5730051 \\
\hline 22. & -.715735 & -.944265 & 28.76601 & 35.12 & -1.092932 & -.7955979 \\
\hline 23. & -.0273541 & -1926459 & 46.63176 & 48.93 & -.341313 & -.0439788 \\
\hline 24. & -.0816869 & -.8483132 & 37.82295 & 47.84 & -.9969802 & -.6996461 \\
\hline 25. & 1.464553 & .145447 & 69.33616 & 78.86 & -.0032201 & .2941142 \\
\hline 26. & .5045084 & -.1845084 & 53.33142 & 59.6 & -.3331755 & -.0358413 \\
\hline 27. & -.1679214 & -.1520786 & 45.39108 & 46.11 & -.3007458 & -.0034115 \\
\hline 28. & .0085355 & .3814645 & 54.19989 & 49.65 & .2327974 & .5301316 \\
\hline 29. & -.7910032 & 1.321003 & 55.93684 & 33.61 & 1.172336 & 1.46967 \\
\hline 30. & -.6235188 & .3335187 & 45.76329 & 36.97 & .1848516 & .4821859 \\
\hline 31. & -.498404 & .688404 & 51.71854 & 39.48 & .5397369 & .8370711 \\
\hline 32. & -.201817 & -.368183 & 42.28939 & 45.43 & -.5168501 & -.2195158 \\
\hline 33. & -.5013949 & -.6186051 & 35.46566 & 39.42 & -.7672722 & -.469938 \\
\hline 34. & .5578442 & -.2378442 & 53.33142 & 60.67 & -.3865113 & - .0891771 \\
\hline 35. & .0977606 & .6622394 & 58.7904 & 51.44 & .5135723 & .8109065 \\
\hline 36. & .1406287 & .1193713 & 52.58701 & 52.3 & -.0292958 & .2680384 \\
\hline 37. & 1.273641 & -.2436406 & 62.14023 & 75.03 & -.3923078 & -.0949735 \\
\hline 38. & -.96746 & -.84254 & 26.90499 & 30.07 & - . 9912071 & -.6938729 \\
\hline 39. & .1630597 & -.2730597 & 47.99651 & 52.75 & -.4217268 & -.1243925 \\
\hline 40. & -.3114794 & -.9485205 & 33.72871 & 43.23 & -1.097188 & -.7998534 \\
\hline 41. & .5937338 & .4662662 & 62.51243 & 61.39 & .317599 & .6149333 \\
\hline 42. & .0828067 & .3271933 & 54.44803 & 51.14 & .1785262 & .4758604 \\
\hline 43. & .963595 & -.4135951 & 56.18497 & 68.81 & -.5622622 & -.2649279 \\
\hline 44. & .9002901 & - 1902901 & 58.17006 & 67.54 & -.3389573 & -.041623 \\
\hline 45. & .0105292 & .6394708 & 57.42565 & 49.69 & .4908037 & .7881379 \\
\hline 46. & -.6105587 & -1.299441 & 25.66431 & 37.23 & -1.448108 & -1.150774 \\
\hline 47. & .3933507 & -.8833507 & 43.28193 & 57.37 & -1.032018 & -.7346836 \\
\hline 48. & -9181119 & -.741888 & 28.76601 & 31.06 & -.8905551 & -.5932209 \\
\hline 49. & .1496011 & 1.200399 & 66.1104 & 52.48 & 1.051732 & 1.349066 \\
\hline 50. & -.1280442 & .3580442 & 52.21481 & 46.91 & 2093771 & .5067113 \\
\hline 51. & .1226839 & .7573161 & 60.27921 & 51.94 & .608649 & .9059832 \\
\hline
\end{tabular}

\begin{tabular}{|c|c|c|c|c|c|}
\hline st_terr1860 & pop1860 & free1860 & sla 1860 & pct 1860 & ls_sl_ m \\
\hline \multirow{3}{*}{ Alabama } & 964201 & 529121 & 435080 & 45.1 & 74.14134 \\
\hline & & & & 0 & 41.66926 \\
\hline & & & & 0 & 54.24953 \\
\hline Arkansas & 435450 & 324335 & 111115 & 25.5 & 64.87336 \\
\hline California & 379994 & 379994 & 0 & 0 & 40.34091 \\
\hline Colorado Territory & 34277 & 34277 & 0 & 0 & 39.0907 \\
\hline Connecticut & 460147 & 460147 & 0 & 0 & 44.01341 \\
\hline Delaware & 112216 & 110418 & 1798 & 1.6 & 48.35159 \\
\hline District of Columbia & 75080 & 71985 & 3185 & 4.4 & 27.93081 \\
\hline Florida & 140424 & 78679 & 61745 & 44 & 65.1715 \\
\hline \multirow{3}{*}{ Georgia } & $1.1 \mathrm{e}+06$ & 595088 & 462198 & 43.7 & 67.35665 \\
\hline & . & . & & 0 & 47.68591 \\
\hline & & & & 0 & 47.92033 \\
\hline Illinois & $1.7 \mathrm{e}+06$ & $1.7 e+06$ & 0 & 0 & 46.98267 \\
\hline Indiana & $1.4 \mathrm{e}+06$ & $1.4 \mathrm{e}+06$ & 0 & 0 & 49.71751 \\
\hline Iowa & 674913 & 674913 & 0 & 0 & 50.7333 \\
\hline Kansas Territory & 107206 & 107204 & 2 & .01 & 50.11089 \\
\hline Kentucky & $1.2 \mathrm{e}+06$ & 930201 & 225483 & 19.5 & 61.22454 \\
\hline Louisiana & 708002 & 376276 & 331726 & 46.9 & 63.14018 \\
\hline Maine & 628279 & 628279 & 0 & 0 & 36.19958 \\
\hline Maryland & 687049 & 599860 & 87189 & 12.7 & 43.37334 \\
\hline Massachusetts & $1.2 \mathrm{e}+06$ & $1.2 \mathrm{e}+06$ & 0 & 0 & 34.16799 \\
\hline Michigan & 749113 & 749113 & 0 & 0 & 45.4199 \\
\hline Minnesota & 172023 & 172023 & 0 & 0 & 39.87208 \\
\hline Mississippi & 791305 & 354674 & 436631 & 55.2 & 74.59766 \\
\hline \multirow{2}{*}{ Missouri } & $1.2 \mathrm{e}+06$ & $1.1 \mathrm{e}+06$ & 114931 & 9.7 & 52.25388 \\
\hline & & & & 0 & 44.63852 \\
\hline Nebraska Territory & 28841 & 28826 & 15 & .01 & 50.18903 \\
\hline Nevada Territory & 6848 & 6857 & 0 & 0 & 51.28027 \\
\hline New Hampshire & 326064 & 326073 & 0 & 0 & 44.87293 \\
\hline New Jersey & 672035 & 672017 & 18 & .01 & 48.62627 \\
\hline \multirow{4}{*}{$\begin{array}{r}\text { New Mexico Territory } \\
\text { New York } \\
\text { North Carolina }\end{array}$} & 93514 & 93514 & 0 & 0 & 42.68506 \\
\hline & $3.9 \mathrm{e}+06$ & $3.9 \mathrm{e}+06$ & 0 & 0 & 38.38745 \\
\hline & 992622 & 661563 & 331059 & 33.4 & 58.64191 \\
\hline & & & & 0 & 53.07745 \\
\hline \multirow[t]{2}{*}{ Ohio } & $2.3 e+06$ & $2.3 e+06$ & 0 & 0 & 49.17054 \\
\hline & & & & 0 & 55.18719 \\
\hline Oregon & 52465 & 52465 & 0 & 0 & 32.99591 \\
\hline Pennsylvania & $2.9 e+06$ & $2.9 \mathrm{e}+06$ & $\theta$ & $\theta$ & 46.27942 \\
\hline Rhode Island & 174620 & 174620 & 0 & 0 & 37.29352 \\
\hline \multirow[t]{2}{*}{ South Carolina } & 703708 & 301302 & 402406 & 57.2 & 70.83913 \\
\hline & & & & 0 & 50.34261 \\
\hline Tennessee & $1.1 \mathrm{e}+06$ & 834082 & 275719 & 24.8 & 58.12107 \\
\hline Texas & 604215 & 421649 & 182566 & 30.2 & 60.82678 \\
\hline Utah Territory & 40273 & 40184 & 89 & .01 & 52.22063 \\
\hline Vermont & 315098 & 315098 & 0 & 0 & 32.21453 \\
\hline Virginia & $1.6 \mathrm{e}+06$ & $1.1 \mathrm{e}+06$ & 490865 & 30.7 & 51.58495 \\
\hline \multirow[t]{2}{*}{ Washington Territory } & 11594 & 11594 & $\theta$ & 0 & 34.16799 \\
\hline & & & & 0 & 57.68761 \\
\hline \multirow[t]{2}{*}{ Wisconsin } & 775881 & 775881 & $\theta$ & $\theta$ & 48.93612 \\
\hline & & & & 0 & 54.01511 \\
\hline
\end{tabular}

Blair T. Johnson \& Christine M. Curley (2021)

Page 19 of 27 


\section{Technical Summary}

\section{Commentary Text}

NB. For final text, see article in Clinical Psychology: Science and Practice (CP:SP), published in 2021. Below is the text submitted to the journal for the article titled, "Divining Structural Factors Related to Intervention Success or Failure: Cultural Sexism versus Other Macro-Level Factors."

As the samples of interventions examined by meta-analyses grow, opportunities grow not just to see whether the interventions succeed but also to understand better what factors may contribute to intervention success (and failure). Early meta-analysts focused mainly on factors assessed directly within the interventions themselves-factors such as population targeted, specific active content, dosage of content, and time lapse at the point of measurement-viz. micro-level factors. Drawing on increasingly more sophisticated conceptual and statistical models, more recent meta-analysts have begun isolating environmental and structural factors-viz. macro-level factors - that may facilitate or inhibit intervention success. Macro-level factors can vary widely across a plethora of possible dimensions, ranging from environmental factors (e.g., pollution, meteorological events) to psychosocial aspects such as intergroup prejudice. The prejudice and discrimination that minorities routinely face, for example, may impede the success of health promotion interventions for such groups. Ecological models are especially germane to thinking about how best to improve individuals' welfare, concentrating on interacting and reciprocal circles of influence that range from intrapsychic to social to societal, micro to macro. Because psychosocial interventions target individuals who subsequently must live in the same environment that plausibly caused the risky or unhealthy profile in the first place, a greater focus on the supportiveness of macrolevel actors would seem crucial.

In this context, Price and colleagues' (in press) recent spatial meta-analysis examined whether the efficacy of psychotherapy for girls might depend on how supportive the cultural milieu is where the therapy took place. Included were 93 therapeutic intervention studies conducted in the United States whose samples included a majority of girls aged four- to 18-years old. The studies were carried out between 1966 and 2017, although most were conducted since the 1990s; they took place in 32 of the 50 states (64\%; there were none in the District of Columbia [DC]). This team utilized secondary data sources comprised of online surveys that evaluate attitudes toward women and men, either directly or indirectly, to gauge the degree of cultural sexism present in each state, and where feasible at the county level. Positive scores are related to greater traditional attitudes or social norms toward women and their gender roles in society, which equates with a normative tendency for both women and men to view women as 


\section{Technical Summary}

having less important social roles, status, and opportunities. Negative scores were coded relative to the nation-level mean and indicate less traditional attitudes or more egalitarian social norms toward women.

Provocatively, in focused analyses, Price et al. (in press) found support for their hypothesis: Over and above micro-level factors examined (e.g., whether an active control was included, mental health problem targeted), psychotherapy interventions conducted in states with lower cultural sexism levels succeeded better than did those conducted in states with higher cultural sexism. In turn, the current article discusses the meaning of cultural sexism and explores other macro-level factors that may also be implicated. A major problem in meta-analysis is that moderator dimensions are almost always correlational and thus investigators must be sophisticated to examine whether alternative factors might be better explanations. To do so, investigators examine multiple-moderator models to statistically control the potential confounders and thus divine what factors are uniquely associated with variation in effect sizes.

\section{Understanding Cultural Sexism}

That levels of cultural sexism as modeled by Price et al. (in press) range so widely across areas in the U.S. is suggestive that attitudes toward women, social roles, gender stereotypes, and gender equality also vary considerably. Price et al. modeled cultural sexism based upon implicit and explicit attitude data, calculating an attitude range that may or may not approximate experienced cultural sexism. Self-reported attitudes may be impacted by rationalization, system justification, and other social desirability biases. Indeed, although implicit attitude scores were strongly correlated with explicit attitudes factored into the cultural sexism construct, implicit attitudes were not as markedly correlated with intervention effect sizes. It should be emphasized that the zero point on Price et al.'s cultural sexism scale represents the average score across the U.S., such that positive scores were above average and negative scores were below it. The mean value of zero probably does not reflect a true neutral point; instead, the U.S. as a whole is culturally sexist, such that even in states with low cultural sexism, girls and women nevertheless experience some amount of cultural sexism. As evidence, consider rampant media objectification of women, including broadcast media, advertising, music, and, social media, which has been shown to influence lower self-esteem, distorted body image, and poorer mental health outcomes for adolescent girls (Grabe \& Hyde, 2009); as well, large percentages of women report experiencing sexual violence in their lifetimes, even in so-called low sexism states (Smith et al., 2017). Nonetheless, we strongly suspect that the range of cultural sexism values that Price et al. documented is real.

In concurrence with Price et al.'s (in press) broader theoretical model emphasizing cultural sexism, we theorize that cultural tightness-looseness is also related to therapeutic success for intervention 


\section{Technical Summary}

studies. Cultures have been shown to vary meaningfully in terms of tightness-many strongly enforced rules and little tolerance for deviance-versus the reverse, looseness-few strongly enforced rules and more tolerance for deviance (e.g., Harrington \& Gelfand, 2014). According to this ecological model, naturalistic or human-made threats increase a culture's need to enforce strong norms so that members can coordinate socially and survive-whether it is to reduce chaos in areas with high population density, to ameliorate resource scarcity, to coordinate against natural disasters, to fend off territorial threats, or to stop the spread of disease. Harrington and Gelfand created a state-level index of tightness-looseness based on documented practices in each state, such as whether corporal punishment was legal in schools, how severely violations of criminal statutes were punished, how religious a state is, and the ratio of foreigners in its population. None of the practices in their scale directly tracks sexism, but one item does so indirectly, support for same-sex marriage. To characterize the extremes of this index, the four loosest states were California, Oregon, Washington, and DC, and the four tightest were Mississippi, Alabama, Oklahoma, and Arkansas; around the mid-point were Minnesota, Michigan, lowa, and Florida. We thus hypothesized that states with higher cultural sexism also are tighter and that states with lower cultural sexism are looser, expanding on Harrington and Gelfand's findings that tightness and tolerance of gender inequality are correlated.

Accordingly, we created a database with Price et al.'s (in press) values for cultural sexism combined along with other state-level factors, such as tightness, history of slaveholding, sex education inclusiveness, and several control variables (e.g., population taken in the 2010 census). The results we present here are the same whether we weight analyses by state population (or not), even though states with interventions were over three times more populous than those without interventions, $F(1$, 49 )=11.50, $p=.0014, R^{2}=0.19$. (Still, this result helps to show that interventions took place in relatively populous areas as opposed to more rural environments. Urban communities often operate differently than do rural ones, a factor that bears further consideration in future meta-analyses of intervention studies.) Further results report the weighted versions.

As we hypothesized, tighter states were much more likely to have higher levels of cultural sexism, $r(49)=.79, p<.0001$, a very large effect size. Thus, states with high cultural sexism were likely to be high in tightness, as well, such as Texas, Kentucky, and Tennessee, although the four states with the highest cultural sexism had no interventions (Mississippi, Alabama, Arkansas, West Virginia). On the other end of the cultural sexism continuum, of the five states with lowest values, DC, Vermont, Oregon, Massachusetts, and Washington, all had interventions except DC; these can all be characterized as quite culturally flexible, "loose" to "very loose." 


\section{Technical Summary}

Given that communities characterized by negative attitudes and stereotypes regarding one minority group tend to have similarly negative views of other minority groups, we were led to posit that more sexist cultures may also hold negative racial views. Payne and colleagues (2019) showed that the degree to which U.S. counties held slaves, as documented in the 1860 U.S. census, was markedly associated with the extent to which Whites' attitudes were negative attitudes toward Blacks ( $r=.64$, $p<.001)$, as gauged in one of Price et al.'s main data sources, Project Implicit. Logically, racism has persisted across a century and a half because of factors associated with slaveholding and its aftermath (e.g., redlining, residential segregation, cultural traditions). Our analysis revealed that this same variable, 1860 slaveholding at the state level, also markedly associates with Price et al.'s cultural sexism values, $r(49)=.63$, $p<.001$, again a large effect size. Hence, yet another factor appears plausibly associated with U.S. clinical intervention success for girls, although because we did not have access to Price et al.'s effect size data we could not make a formal test. Gender and race are frequently linked in contemporary treatments of intersectionality; thus, macro-level factors may well be baked into the societal mix.

Because Price et al.'s (in press) attitudinal index of cultural sexism aligns so closely with the tightness-looseness index, which is based on more formal variables, community attitudes and norms may be intertwined with policies within individual states, including laws and enforcement of those laws. Yet, because this work is correlational, it is important to note that causality may reverse: Attitudes can conform to local policies after the fact, perhaps because preservation of the status quo in tighter states may block changes in social norms toward greater gender equality. A more idealistic perspective is that networks of individuals develop attitudes supportive of gender equity and that these attitudes then drive political change that institutionalizes equitable treatment under the law. Either way, cultural sexism would seem to be much more interwoven into the fabric of other dimensions than its label would indicate.

The range of culturally sexist attitudes that Price et al. (in press) documented also may be associated with state-level sex education mandates and specific required components of the curriculum. Research evaluating sex education in the United States and other nations over the past 30 years has found that comprehensive sex education-especially when required to be medically accurate and include curricula such as birth control, safe sex, consent, refusal, boundaries, healthy relationships, and sexual decision-making, as opposed to focusing primarily on abstinence, preventing pregnancy and sex only in marriage-is not only associated with lower rates of intimate partner violence, unwanted pregnancies, and sexually transmitted infections but also is associated with better socio-emotional learning, interpersonal skills and self-esteem for adolescent girls (Goldfarb \& Lieberman, 2020). Although not specifically examined in the studies reviewed, the connection between these latter influences and the 


\section{Technical Summary}

psychological health of young girls is well-established in the literature. We compiled an index of sex education inclusiveness, aggregating the various components of sex education curriculum such as contraception, healthy relationships, refusal, and consent-from the Guttmacher Institute's (2021) extensive review of state-level mandates. States with higher more cultural sexism also had less inclusive curricula, $r(46)=-.55, p<.001$. (The same association emerged with cultural tightness, $r(46)=-.63, p<.001$.)

A puzzle from Price et al.'s (in press) results is that, although state-level cultural sexism was associated with intervention efficacy for adolescent girls, the same pattern did not emerge with countylevel cultural sexism as a predictor (although interaction effects did, such as less intervention success in states where both county level and state level cultural sexism were high). On the face of it, this finding appears to contradict the first law of geography, that more proximal factors have a greater influence on outcomes than those farther away. Yet, we posit that this finding reflects the practical difficulties of more micro factors countering state-level mandates effectively. For example, in Mississippi, a state that is both culturally tight and culturally sexist, localities may include only instruction about contraception and prevention of sexually transmitted infections when they have the express permission of the state-level Department of Education. Similarly, in Utah, teachers are prohibited from answering student questions with information that might conflict with state sex education laws, which must stress abstinence and that sexual intercourse takes place only in marriage.

The same conclusion emerges from another marker of cultural sexism, the protection-or lack thereof-of women who have been victims of sexual violence or intimate partner assault. This factor is also dependent on state-level laws. Thus, the four tightest states-Alabama, Arkansas, Mississippi, and Oklahoma, which scored high on cultural sexism and lacked studies evaluating psychotherapy interventions for girls-also have no employment rights laws providing intimate partner violence victims with leave from work or broader protections against employment discrimination related to the violence (statusofwomendata.org, 2021). These states also elevate protecting gun rights over protecting women from future violence, failing to enact laws to prohibit gun possession by persons convicted of domestic or sexual violence, or subject to domestic violence protective orders, and do not require removal of firearms from such individuals. One would be hard-pressed to envision county-level efforts that could ameliorate state-level policy decisions such as these. Hence, even when localities are less sexist (e.g., teachers, health-care providers, government officials), their views can run afoul of a more powerful network, which in turn squelches efforts to act differently. Similarly, major social norm changes, such as recognition of same-sex marriage, although effectuated through some state-level policy changes, remained scattershot 


\section{Technical Summary}

until the U.S. Supreme Court decision in Obergefell v. Hodges (2015) 135 S.Ct. 2071, 576 U.S. 644, permitted same-sex marriages as the law of the land.

Finally, at their most potent, media both traditional and social easily cut across traditional spatial boundaries; real or imagined events broadcast swiftly through networks and can harm networks of related individuals such as sexual minorities. By the same token, some actions-such as promoting social clubs for stigmatized individuals - have been shown to create resilience in affected adolescents. As an example, Hatzenbuehler and colleagues (2019) documented that homophobic bullying of school children increased in California after a state-wide referendum restricted marriage to heterosexuals; importantly, this pattern was restricted to schools where there were no gay-straight alliances. Thus, allies across social lines could help ameliorate an important problem.

\section{Concluding Remarks}

Price and colleagues (in press) have provided valuable preliminary evidence that a dimension of cultural sexism can countervail efforts for psychotherapy to succeed in samples that focus on girls aged four to 18. Our own examination reveals cultural sexism to be associated with at least three macro-level factors: cultural tightness, historical slaveholding (and by implication racism), and sex education inclusiveness. The fact that cultural sexism can be so well predicted by these factors is additional evidence that cultural sexism is real, yet it also suggests caution in interpreting these effects as merely reflecting cultural sexism. Surely, the reality is more complex. Thus, we believe that understanding effects of interventions at the macro level requires a more extensive model, one that incorporates objective measures of sexism beyond markers such as income, income inequality, poverty, and education, and meaningfully theorizes about how such dimensions might interact. For example, sexism is logically more pernicious to the extent that a culture is tight; nation-level changes such as same-sex marriage would seem to have considerable potential to improve mental health for affected individuals; finally, media avenues also are a potentially extremely powerful force as these easily cross artificial spatial boundaries. Our findings further suggest that understanding the structural policy components of cultural sexism, of which this essay is merely a beginning, could inform future interventions to improve the psychological health outcomes for adolescent girls. Along these lines, the same meta-analytic framework could be used to assess the success of psychotherapy interventions not only for girls but also boys and others, especially those at the intersection of stigmatized identities (e.g., sexual minorities of color). The results from such models promise to point the way to improved therapies.

As a final note, consider again that all of the factors we have discussed here are correlational. The very factors that appear to undercut therapeutic success may be the factors that make individuals more 


\section{Technical Summary}

susceptible to mental health problems in the first place. Individuals have needs left wanting or even worsened by the local cultures that envelope them, a prediction that Johnson and colleagues' (2010) ecological model makes. Thus, interventions might succeed in the sense that a young person comes to develop self-worth and perhaps even to experience lower anxiety levels. An intervention might thus succeed in the very short term-because needs are so deep-yet fail in the long run because surrounding networks are so strongly countervailing. If a troubled young girl's community is stuck in the mud, can she ever free herself of its stains? 


\section{Technical Summary}

\section{References}

Goldfarb, E. S., \& Lieberman, L. D. (2020). Three decades of research: The case for comprehensive sex education. Journal of Adolescent Health, doi:10.1016/j.jadohealth.2020.07.036

Grabe, S., \& Hyde, J. S. (2009). Body objectification, MTV, and psychological outcomes among female adolescents. Journal of Applied Social Psychology, 39(12), 2840-2858. doi:10.1111/j.15591816.2009.00552.x

Guttmacher Institute. (2021). Sex and HIV education. Retrieved from https://www.guttmacher.org/statepolicy/explore/sex-and-hiv-education\#

Harrington, J. R., \& Gelfand, M. J. (2014). Tightness-looseness across the 50 united states. Proceedings of the National Academy of Sciences of the United States of America, 111(22), 7990-7995. doi:10.1073/pnas.1317937111 [doi]

Hatzenbuehler, M. L., Shen, Y., Vandewater, E. A., \& Russell, S. T. (2019). Proposition 8 and homophobic bullying in california. Pediatrics, 143(6), 10.1542/peds.2018-2116. Epub 2019 May 13. doi:e20182116 [pii]

Johnson, B. T., Redding, C. A., DiClemente, R. J., Mustanski, B. S., Dodge, B., Sheeran, P., ... Fishbein, M. (2010). A network-individual-resource model for HIV prevention. AIDS and Behavior, 14(Suppl 2), 204-221. doi:10.1007/s10461-010-9803-z [doi]

Payne, B. K., Vuletich, H. A., \& Brown-lannuzzi, J. L. (2019). Historical roots of implicit bias in slavery. Proceedings of the National Academy of Sciences of the United States of America, 116(24), 1169311698. doi:10.1073/pnas.1818816116 [doi]

Price, M. A., McKetta, S., Weisz, J. R., Ford, J. V., Lattanner, M. R., Skov, H., . . Hatzenbuehler, M. L. (in press). Cultural sexism moderates efficacy of psychotherapy results from a spatial meta-analysis. Clinical Psychology: Science and Practice.

Smith, S. G., Basile, K. C., Gilbert, L. K., Merrick, M. T., Patel, N., Walling, M., \& Jain, A. (2017). National intimate partner and sexual violence survey (NISVS): 2010-2012 state report.

statusofwomendata.org. (2021). Violence \& safety. Retrieved from https://statusofwomendata.org/explore-the-data/violence-safety/ 